\title{
Characterization of Partitioning in a Medium-Mn Third-Generation AHSS
}

\author{
J T Benzing ${ }^{1}$, J Bentley ${ }^{2}$, J R McBride ${ }^{3}$, D Ponge ${ }^{4}, \mathrm{~J} \mathrm{Han}^{5}$, D Raabe ${ }^{4}, \mathrm{~J} \mathrm{E} \mathrm{Wittig}^{1}$ \\ ${ }^{1 .}$ Interdisciplinary Materials Science, Vanderbilt University, Nashville TN 37232, USA \\ 2. Microscopy and Microanalytical Sciences, PO Box 7103, Oak Ridge, TN 37831-7103, USA \\ 3. Vanderbilt Institute of Nanoscale Science and Engineering, Vanderbilt University, Nashville TN 37232, USA \\ 4. Max-Planck-Institut für Eisenforschung, Max-Planck-Straße 1, D-40237 Düsseldorf, Germany \\ 5. Materials Science and Engineering, Chungnam National University, Daejeon 34134, Republic of Korea
}

Medium-Mn steels are considered third generation advanced high strength steels (AHSS), which contain both ferrite $(\alpha)$ and austenite $(\gamma)$ and aim to balance the low cost of first generation AHSS with the outstanding mechanical properties of second generation AHSS. These steels generally exhibit an $\alpha^{\prime}$ martensitic microstructure after hot and cold rolling but after inter-critical annealing have a dual-phase microstructure of $\alpha$ with a volume fraction of reverted $\gamma$ that ranges between 20 and 50\%. Mechanical properties of a third generation AHSS ideally exhibit low strain hardening rates at low levels of strain and higher strain hardening rates as total strain accumulates [1]. Studies investigating deformation mechanisms and mechanical properties of medium-Mn steels have reported the effects of inter-critical annealing temperature and phase fractions/sizes [2-5]. Cold-rolling medium-Mn steels prior to inter-critical annealing is necessary to retain $\gamma$ [6]. The partitioning of $\mathrm{Al}$ from $\gamma$ to $\alpha$ and partitioning of $\mathrm{Mn}$ and $\mathrm{C}$ from $\alpha / \alpha^{\prime}$ to $\gamma$ during inter-critical annealing in the $\gamma+\alpha$ phase field controls both the amount and stacking fault energy (SFE) of the reverted $\gamma$. The SFE is important for transformation and twinning-induced plasticity (TRIP/TWIP). Characterizing partitioning in this multi-phase alloy will aid in interpreting the mechanical properties of the constituent phases. This study utilizes transmission electron microscopy (TEM), convergent beam electron diffraction (CBED) and scanning TEM energy-dispersive X-ray spectroscopy (STEM-EDS) performed using an FEI Tecnai Osiris equipped with a quad Super-X detector to characterize partitioning among the ultrafine-grained (UFG) phases in a Fe-12Mn-3Al-0.06C steel.

An ingot of Fe-12Mn-3Al-0.06C (wt\%) was hot-rolled at $900^{\circ} \mathrm{C}$ to $3.4 \mathrm{~mm}$ thickness, homogenized in an $\mathrm{Ar}$ atmosphere at $1100^{\circ} \mathrm{C}$ for $2 \mathrm{~h}$, water-quenched to room temperature (RT) and cold-rolled to $1.7 \mathrm{~mm}$. Thermo-Calc software predicts an equilibrium $\gamma$ volume fraction of 0.45 , a $\gamma$ composition of Fe-20Mn-2.2Al-0.12C and a RT $\mathrm{SFE} \sim 20 \mathrm{~mJ} / \mathrm{m}^{2}$ following inter-critical annealing at $585^{\circ} \mathrm{C}$. To study the effect of annealing time on phase fraction, samples were annealed for 0.5 and $8 \mathrm{~h}$. Punched 3-mm discs were electropolished to electron transparency with a Struers twin-jet system $\left(5 \% \mathrm{HClO}_{4}, 35 \%\left[\mathrm{CH}_{3}\left(\mathrm{CH}_{2}\right)_{3}\right]-\mathrm{O}-\mathrm{C}_{2} \mathrm{H}_{4} \mathrm{OH}, 60 \% \mathrm{CH}_{3} \mathrm{OH}\right.$ by volume, $\left.-30^{\circ} \mathrm{C}, 15 \mathrm{~V}\right)$. Bruker Esprit software was used to record $200 \mathrm{kV}$ STEM-EDS hypermaps with typically $\sim 40 \mu \mathrm{s}$ dwell, $2-3 \mathrm{~nm}$ pixels, $\sim 2 \times 2 \mu \mathrm{m}$ scanned area and $\sim 20$ frames for $\sim 600 \mathrm{~s}$ acquisition. Processing included background subtraction, deconvolution and normalized wt\% mapping. Data were also acquired with FEI TIA software. As seen in Figure 1, a sample annealed for $0.5 \mathrm{~h}$ contained mostly $\alpha$, but also a decoration of Mn-enriched $\gamma$. CBED analysis was successful for samples annealed for $8 \mathrm{~h}$ [Figure 2(a-c)], but was too challenging for material aged for $0.5 \mathrm{~h}$. In Figure 2(d), a processed line scan supports the Thermo-Calc predictions of $\gamma$ phase enriched in Mn and depleted in Al. As $\gamma$ grains were preferentially electropolished, $\mathrm{Mn} / \mathrm{Fe} \mathrm{K}_{\alpha}$ peak ratios from spot analyses are useful for phase identification [e.g. Figures 1(a) and 3(a,e)]. Quantitative STEM-EDS mapping of samples annealed for 8 $\mathrm{h}$ at $585^{\circ} \mathrm{C}$ [Figure 3(b-d, f-h)] revealed a $\gamma$ phase enriched to $\sim 20 \mathrm{wt} \% \mathrm{Mn}$ and depleted to $\sim 1.3 \mathrm{wt} \% \mathrm{Al}$, thus matching the equilibrium Thermo-Calc predictions. Since quantifying carbon by STEM-EDS is impossible for the levels of interest, a concurrent atom probe tomography study is underway, in part to measure $\mathrm{C}$ partitioning. FIB lift-out specimens that mitigate the limitations imposed by the selective electropolishing and the ferromagnetism of self-supporting discs should allow composition profiles at (edge-on) phase boundaries to be obtained [7].

[1] D K Matlock and J G Speer, pp. 185-205 in Microstructure and Texture in Steels, ed A Halder, S Suwas and D Bhattacharjee (Springer, London), 2009.

[2] S Lee and B C De Cooman, Metall. Mater. Trans. A 44 (2013) 5018-5024.

[3] M M Wang, C C Tasan, D Ponge, and D Raabe, Acta Mater. 111 (2016) 262-272.

[4] Y-K Lee and J Han, Mater. Sci. Technol. 31 (2015) 843-856.

[5] S Lee and B C De Cooman, Metall. Mater. Trans. A 45 (2014) 6039-6052.

[6] J Han et al., Acta Mater. 122 (2016) 199-206. 
[7] This work was funded by the US National Science Foundation, Division of Materials Research under grant DMR1309258, NSF EPS 1004083 and the MPIE in Düsseldorf, Germany.

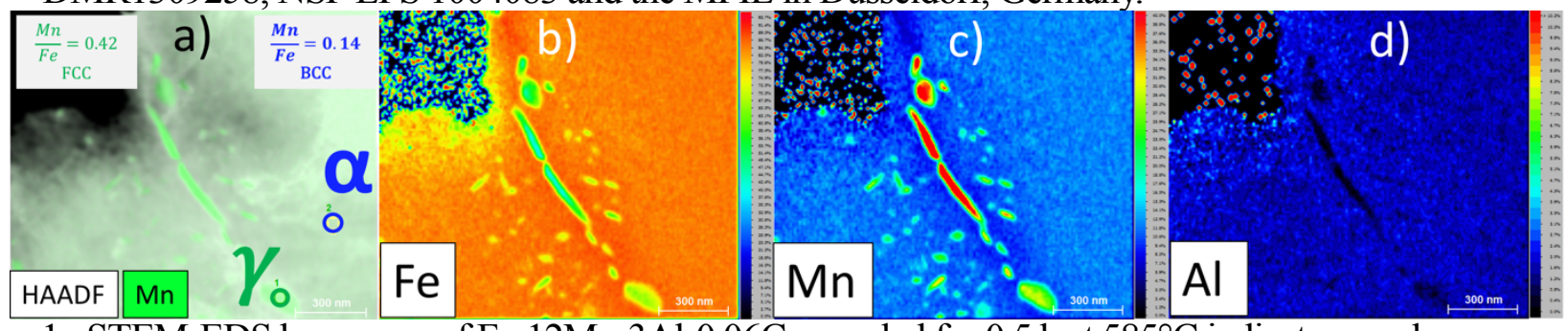

Figure 1: STEM-EDS hypermap of Fe-12Mn-3Al-0.06C annealed for $0.5 \mathrm{~h}$ at $585^{\circ} \mathrm{C}$ indicates a $\gamma$ phase enriched in $\mathrm{Mn}$ and depleted in Fe and $\mathrm{Al}(39 \mu$ s dwell, $2 \mathrm{~nm}$ pixels, $1.6 \times 1.3 \mu \mathrm{m}$ scanned area, 19 frames). (a) Spot-EDS from marked locations distinguishes between $\alpha$ and $\gamma$ phases via $\mathrm{Mn} / \mathrm{Fe} \mathrm{K}_{\alpha}$ peak ratios. Quantitative maps for (b) 0-95 wt \% Fe, (c) 0-40 wt \% Mn, and (d) 0-10 wt $\% \mathrm{Al}$.
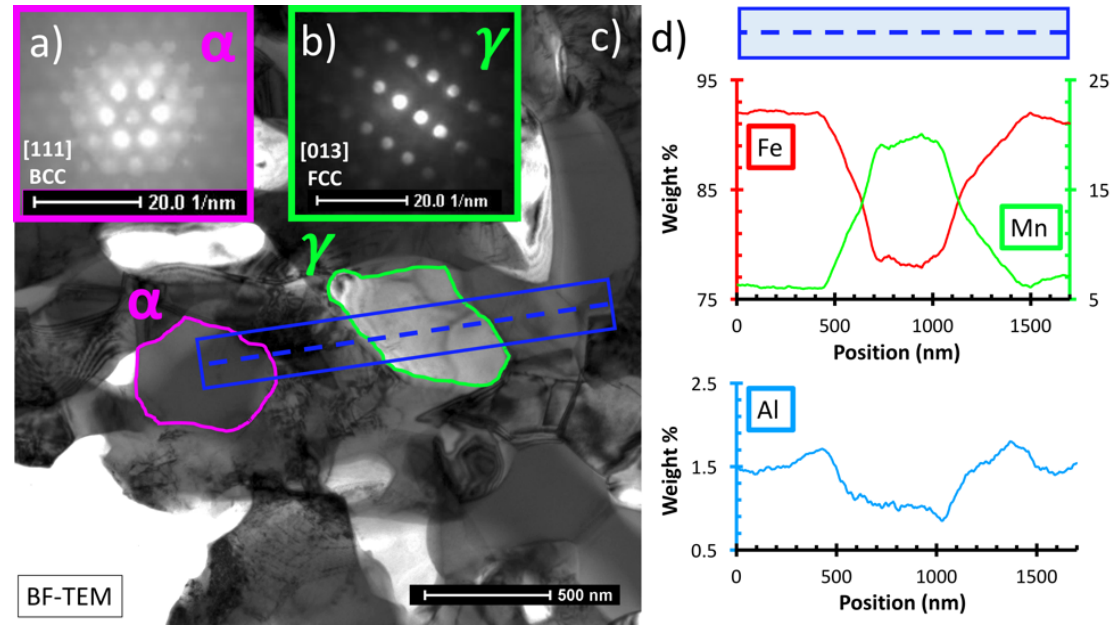

Figure 2: Fe-12Mn-3Al-0.06C annealed for $8 \mathrm{~h}$ at $585^{\circ} \mathrm{C} .200 \mathrm{kV} \mathrm{CBED}$ patterns used to identify (a) ferritic $(\alpha)$ and (b) austenitic $(\gamma)$ grains shown in (c) the bright-field TEM image. (d) Line scan [position marked on (c)], extracted from hypermap, across $\alpha$ and $\gamma$ grains, highlights normalized differences in $\mathrm{Fe}, \mathrm{Mn} \& \mathrm{Al}(\mathrm{wt} \%$ ).

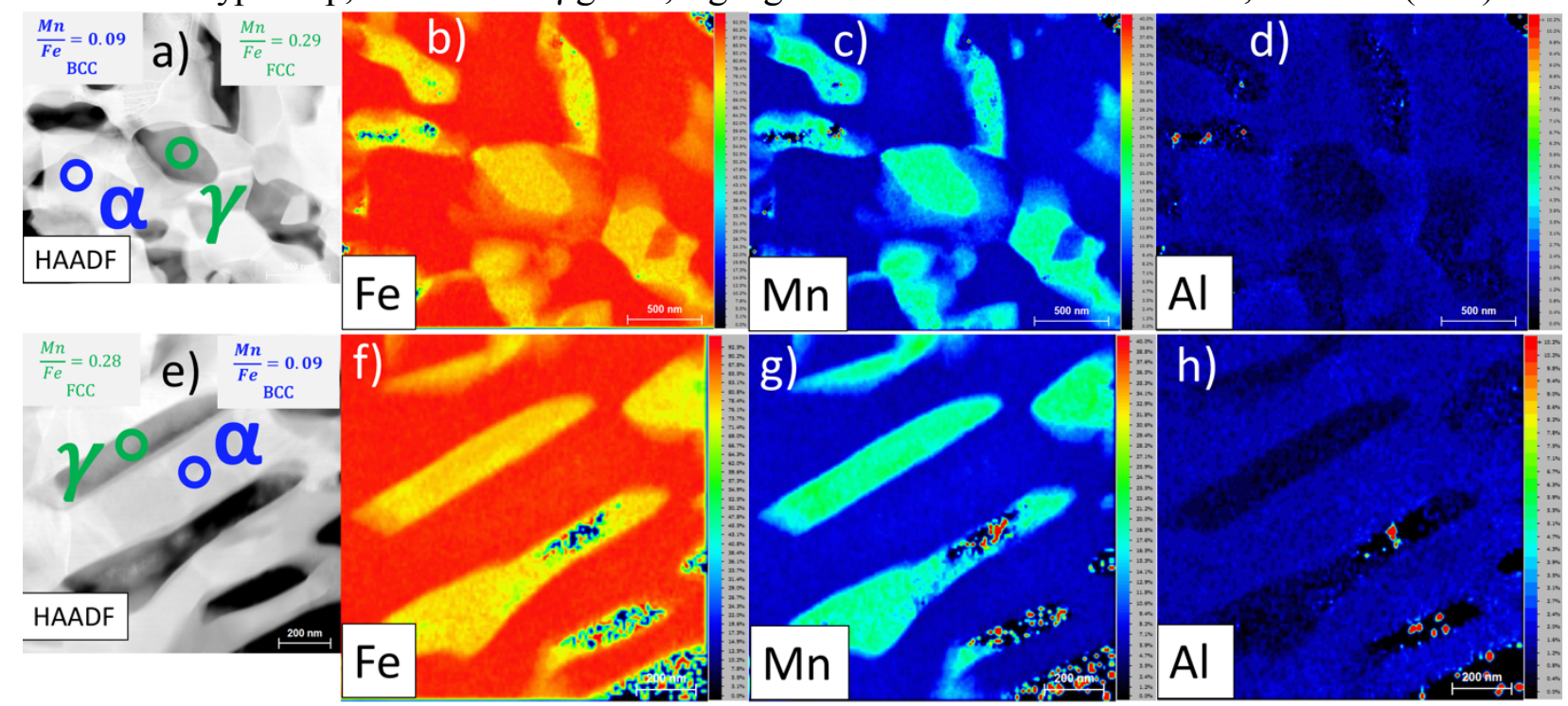

Figure 3: STEM-EDS hypermaps of Fe-12Mn-3Al-0.06C annealed for $8 \mathrm{~h}$ at $585^{\circ} \mathrm{C}$ indicates a $\gamma$ phase enriched in $\mathrm{Mn}$ and depleted in Fe and Al. (a-d) $39 \mu \mathrm{s}$ dwell, 3-nm pixels, 2.5 × $2.1 \mu \mathrm{m}$ scanned area, 35 frames, (e-h) $34 \mu \mathrm{s}$ dwell, 2-nm pixels, $1.2 \times 1.2 \mu \mathrm{m}$ scanned area, 19 frames. (a,e) Spot-EDS from marked locations identifies $\gamma$ and $\alpha$ phases via $\mathrm{Mn} / \mathrm{Fe} \mathrm{K}_{\alpha}$ peak ratios. Quantitative maps for $(\mathrm{b}, \mathrm{f}) 0-95 \mathrm{wt} \% \mathrm{Fe},(\mathrm{c}, \mathrm{g})$ 0-40 wt\% Mn, (d,h) 0-10 wt $\% \mathrm{Al}$. 